RESEARCH ARTICLE

\title{
Who sleeps better? Sleep patterns and sleep disturbances in adolescents with recurrent abdominal pain or inflammatory bowel disease and healthy controls
}

\author{
Ann-Kristin Manhart ${ }^{*} \quad$ Angelika A. Schlarb $^{1}$ \\ ${ }^{1}$ Faculty for Psychology and Sports, Bielefeld University, 33015 Bielefeld, North-Rhine Westphalia, Germany
}

\section{Check for updates}

Correspondence to: Ann-Kristin Manhart, Department of Psychology and Sports, Clinical Psychology and Psychotherapy for Children and Adolescents, Bielefeld University, 33501 Bielefeld, Germany; E-mail: ann-kristin.manhart@uni-bielefeld.de

Received: July 13, 2021;

Accepted: August 27, 2021;

Published: August 31, 2021

Citation: Manhart AK and Schlarb AA. Who sleeps better? Sleep patterns and sleep disturbances in adolescents with recurrent abdominal pain or inflammatory bowel disease and healthy controls. Theory Clin Pract Pediatr, 2021, 3(1): 83-93.

https://doi.org/10.25082/TCPP.2021.01.005

Copyright: () 2021 Ann-Kristin Manhart and Angelika A. Schlarb. This is an open access article distributed under the terms of the Creative Commons Attribution License, which permits unrestricted use, distribution, and reproduction in any medium, provided the original author and source are credited.

\begin{abstract}
Background: Sleep difficulties play an important role in the maintenance and course of chronic abdominal pain disorders (RAP and IBD). Particularly among adolescents with inflammatory bowel diseases (IBD) or recurrent abdominal pain (RAP), adequate sleep seems to be important, as the diseases self and the associated symptoms can cause distress and impair daytime functioning. Hence it seems adequate to take a closer look concerning the sleep difficulties within the different conditions of abdominal pain especially in comparison to a healthy control. To our knowledge no former study compared sleep problems in youths with RAP and IBD as well as healthy controls. Thus the aim of the present study was to 1) evaluate sleep problems in the RAP and IBD and 2) compare the sleep problems of these abdominal pain diseases with a healthy control group. Methods: 129 adolescents (14-25 years) took part in the online survey, with 58 suffering from IBD, 23 had RAP and 48 healthy controls. Adolescents completed sleep questionnaires as PSQI, SDSC or NEQ. Data was analysed by conducting MANOVAs to test differences between the three groups followed by a post-hoc analyses. Results: Significant differences between both patient groups and healthy controls regarding sleep quality as well as sleep disturbances were found. Results indicate that especially young IBD patients suffered more often from poor sleep quality, sleep disturbances as well as daily effects of nightmares than the control group. The comparison of adolescents with RAP and healthy controls showed elevated scores concerning sleep disturbances for RAP patients. However, IBD and RAP adolescents did not differ significantly concerning most sleep measurements. Discussion: The study at hand was the first to compare adolescents with IBD and RAP regarding sleep difficulties. Adolescents with IBD and RAP have an impaired sleep quality as well as a higher rate of sleep disturbances and suffer from daily effects of nightmares than the control group. Therefore sleep disturbances should be also addressed when treating IBD and RAP patients to prevent further impairments.
\end{abstract}

Keywords: IBD, RAP, sleep, insomnia, nightmares, daytime consequences

\section{Background}

With prevalence rates, increasing with age, between 9 and 25\% RAP is a common syndrome in children and adolescents [1]. In fact $15 \%$ of pediatric visits are due to abdominal pain without organic causes [2]. RAP is diagnosed if at least three episodes of abdominal pain with in a three month period have been reported, without identifying a medical reason and when the pain is associated with the use of health care services and/or impairment in daily life (e.g. school attendance) [3]. Patients with RAP report pain mostly located in the periumbilical region, periodic described as sore, colicky and/or sharp [4]. Studies concerning school-aged children show a prevalence of weekly abdominal pain ranging from 11 to $38 \%$ [5-7].

Suffering from chronic pain is associated with various health impairments and therefore often associated with reduced quality of life. Concerning self-reported quality of life young RAP patients report equal scores as children with inflammatory bowel disease (IBD), both show lower scores compared to healthy controls [8]. The etiology of RAP is still not fully understood. However, based on the biopsychosocial model which takes interaction between biological, social and psychological factors into account might explain these symptoms (e.g. emotional stress) [9]. One juncture between biology, psychology and environment is sleep. Hence the association of sleep and chronic abdominal pain is of great theoretical as well as clinical interest. Studies addressing the association of sleep disturbances in children with RAP are rare [10]. However, higher levels of sleep disturbances for young RAP patients compared to healthy controls, especially impairments concerning insufficient sleep, poor sleep quality and 
daytime tiredness were reported in various studies [10-13]. This goes in hand with findings that modification of cognition, attention as well as affect regulation resulting from sleep disturbances seem to play an important role in the ability to cope with pain $[10,14]$.

Characteristically, first onset of IBD is during young adulthood or even earlier in adolescence $[15,16]$ and typically occurring in relapses $[17,18] .25 \%$ of the affected people are younger than 20 years, with a peak in adolescence [18-20]. An early beginning of IBD often appears with a more aggressive form of the disease as well as more complications (e.g. poor nutrient supply leading to insufficient growth) [21-23]. Further, an early onset of IBD is accompanied by other health impairments as well as deficiencies regarding quality of life (e.g. diarrhea, fever, cramps, tiredness, weight loss and a general feeling of sickness) [24-26]. Those circumstances make it an even bigger challenge for younger IBD patients to master age-oriented developmental tasks during puberty [27]. It seems that impaired sleep has an enormous impact on the course of the disease e.g. provoking an episode of IBD symptoms and vice versa increased disease activity might lead to sleep disturbances and poor sleep quality [28,29]. Therefore, this association appears also to be bidirectional [28]. Beyond, sleep problems could be provoked by medical treatment (corticosteroids), disease symptoms and disease-related immune changes [30].

Even though nightmares are a frequent sleep problem during childhood [31], there are rarely studies concerning the association of chronic pain and nightmares in children and adolescents. Pirinen and collegues (2010) reported that children and adolescents with inflammatory bowel disease (IBD) experience more nightmares compared to their healthy controls [32]. To our knowledge no further data exists concerning nightmares and especially its daily effects in children and adolescents with chronic abdominal pain.

Obviously sleep problems play an important role in the maintenance and course of chronic abdominal pain disorders (RAP and IBD), therefore it appears inevitably to take a closer look concerning its association with the different conditions of abdominal pain. To our knowledge no former study investigated the differences of sleep problems in youths with RAP and IBD as well as healthy controls. Thus the aim of the present study was to (1) evaluate sleep problems in the RAP and IBD and (2) compare the sleep problems of these abdominal pain diseases with a healthy control group.

\section{Methods}

\subsection{Procedure}

Various hospitals in Germany, mostly with specialized pediatric departments for children and adolescents with gastrointestinal diseases as well as pediatric practices were contacted and requested to lay open flyers containing information about the online study and a link to the survey as well as a QR-Code for their patients. This study was an online survey created with the program Unipark of the EFS Survey Software (QuestBack AG, 2015) [33]. The survey lasted 15 - 20 minutes. Furthermore, the link of the online survey was dispersed via online forums for IBD patients and their relatives in social networks. It contained self as well as parental ratings. To include healthy participants as controls, the study link was also distributed via E-Mail and social networks. To keep the discontinuation rate as low as possible all items had to be filled out in order to proceed with the survey. 2318 adolescents and young adults between 14 and 25 years visited the study. After excluding data of participants who withdraw from the study or did not complete the online survey, a number of 159 adolescents remained. Further 30 had to be excluded due to inconclusive statements concerning their diagnosis, hence a number of 129 participants remained. All participants had to give their informed consent before they could start the survey. They were informed that participation is voluntary and there is the possibility to withdraw from the study at any time without any consequences. Ethics approval for the research project was obtained from the ethics committee of Bielefeld University.

\subsection{Participants}

Inclusion criteria for the pain group were either a diagnosed Crohn's disease (CD) or Ulcerative colitis (UC) since at least 6 months or recurrent abdominal pain for at least 6 months and also healthy adolescents as control condition (CC). In sum, 2318 adolescents and young adults 14 to 25 years started the in study. Therefore, 2189 data of participants had to be excluded due to not completing the survey or withdrawing from the study, as well as those not meeting inclusion criteria, with a remaining number of 129 adolescents. Further 30 had to be excluded due to inconclusive statements concerning their diagnosis, hence 129 participants remained. Of those, 23 had RAP (age (years) $M=22.7 ; S D=2.2 ; 91.3 \%$ female), 58 suffered from IBD (age (years) $M=20.5 ; S D=3.49 ; 79.3 \%$ female) and 48 were healthy controls (age (years) $M=$ 
23.4; $S D=1.3 ; 78.6 \%$ female). There was a significant difference concerning age between the youths suffering from IBD compared to young RAP patients $(\mathrm{p}=.004)$ as well as compared to the healthy controls $(\mathrm{p}=0.000)$. Characteristics of the sample are shown in Table 1 .

Table 1 Sample characteristics

\begin{tabular}{lccccccc}
\hline \multirow{2}{*}{ Variable } & \multirow{2}{*}{$\begin{array}{c}\text { age (years) } \\
\text { M (SD) }\end{array}$} & \multicolumn{2}{c}{ gender } & & \multicolumn{3}{c}{$\mathrm{N}(\%)$} \\
\cline { 3 - 4 } \cline { 7 - 8 } & & female & male & & Crohn's disease & Colitis ulcerosa \\
\hline RAP & $22.7(2.2)$ & $2(34.5 \%)$ & $21(65.5 \%)$ & & 23 & \\
IBD & $20.5(3.5)$ & $12(20.7 \%)$ & $46(79.3 \%)$ & & $40(69 \%)$ & & $18(31 \%)$ \\
Controls & $23.4(1.3)$ & $9(21.4 \%)$ & $33(78.6 \%)$ & & 42 & \\
\hline
\end{tabular}

\subsection{Measures}

\subsubsection{Sociodemographic data}

Before answering sleep-related questions, general information concerning age, sex, weight and job (pupil, student, apprentice or employed person) of the participants were gathered (4 items). If they suffered from RAP or IBD (diagnosis by pediatricians), participants had to rate duration and intensity of the current symptoms on a visual analog scale from $0-10$. These two questions were only presented to those individuals reporting to be diagnosed with RAP or IBD.

\subsubsection{Self-rating: Pittsburgh Sleep Quality Index (PSQI)}

Sleep and sleep-related problems were rated by the adolescents with the Pittsburgh Sleep Quality Index (PSQI) [34]. The PSQI measures sleep quality (e.g. sleep onset latency, sleep duration, time of waking up and going to bed), during the previous 4 weeks and consists of seven subscales (sleep duration, sleep disturbance, sleep latency, daytime dysfunction, habitual sleep efficiency, subjective sleep quality and sleep medication usage) as well as the global PSQI score. The PSQI global score can range between 0 and 21, with higher scores indicating worse sleep quality. The total score of the PSQI can be divided into three subranges concerning sleep quality: a total score 0-5 equals "good" sleep quality, a total score 6-10 equals "poor" sleep quality and a clinical relevant "disturbed" sleep quality is indicated at a total score $>10$ [34-37]. Internal homogeneity of the PSQI was high with Cronbach's $\alpha=0.83$ ) [34]. Backhaus et al (2002) found high overall PSQI test-retest reliability $(r=0.87)$ as well as modest to high test-retest reliabilities for all seven components $(r=0.53$ to $r=0.88)$ [38].

\subsubsection{Nightmare Effect Questionnaire (NEQ)}

In order to measure the appearance of nightmares the Nightmare Effect Questionnaire (NEQ) was used [39]. The NEQ contains two sections. The first section assesses the frequency of different types of dreams via 6 items. The second part scales the daily impairments due to nightmares with 35 items. The NEQ consists of 6 factors (emotional regulation, stress and aggression, depressive symptoms, attention and concentration, anxiety symptoms, hyperactivity) with a reliability of $\alpha=0,929$ for the global score.

\subsubsection{Parental rating: Sleep Disturbance Scale for Children (SDSC)}

Parents filled in the sleep disturbances the Sleep Disturbance Scale for Children SDSC [40]. The questionnaire consists of 26 items in a Likert-type rating scale with a cut-off score of 39. It contains six subscales (disorders of initiating and maintaining sleep, sleep breathing disorders, disorders of arousal/nightmares, sleep wake transition disorders, disorders of excessive somnolence, sleep hyperhydrosis). Internal consistency was high in controls (0.79) and at a satisfactory level in clinical subjects $(0.71)$; the test/re-test reliability was sufficient for the total score $(0.71)$ as well as the single item scores [40].

\subsection{Data analysis}

Prior to analysis, data for all variables were inspected for missing values. Since the sample was large enough only complete cases were included in order to prevent bias. Data was analysed with the Statistical Package for Social Sciences version 22 (IBM, 2012). Each variable was tested for normal distribution (Shapiro-Wilk-Test). All variables were normally distributed. Hence a MANOVA was conducted in order to test differences in between IBD patients, RAP sufferers and healthy controls. Multivariate analyses of variances (MANOVAs) were conducted to examine differences between the three diagnosis groups (RAP, IBD, controls) and the different sleep 
measurements. The dependent variables were sleep quality (PSQI), sleep disturbances (SDSC) and nightmares (NEQ). For post-hoc analyses a Bonferroni adjusted tests were performed. The significance level was set to $\alpha=0.05$, a tendency of significance was detected at $\mathrm{p}<0.10$. All calculated effect sizes were interpreted according to the guidelines proposed by Cohen (1988) [41].

\section{Results}

According to the PSQI total score, only $18.7 \%$ of the adolescents reported a "good" sleep quality, $58.5 \%$ stated a "poor" sleep quality and $22.8 \%$ indicated to have a "disturbed" sleep quality. Following a Multivariate analysis of variances for the three sample groups was conducted to test if they differed significantly from each other concerning sleep quality. A significant MANOVA effect was obtained, $V=0.283, \mathrm{~F}(14,230)=2.712, p=0.001$. Therefore, Bonferroni adjusted Post-hoc tests were performed to examine individual mean difference comparisons for the three groups and significant factors. Subscale mean values of the PSQI are displayed in Figure 1 and 2.

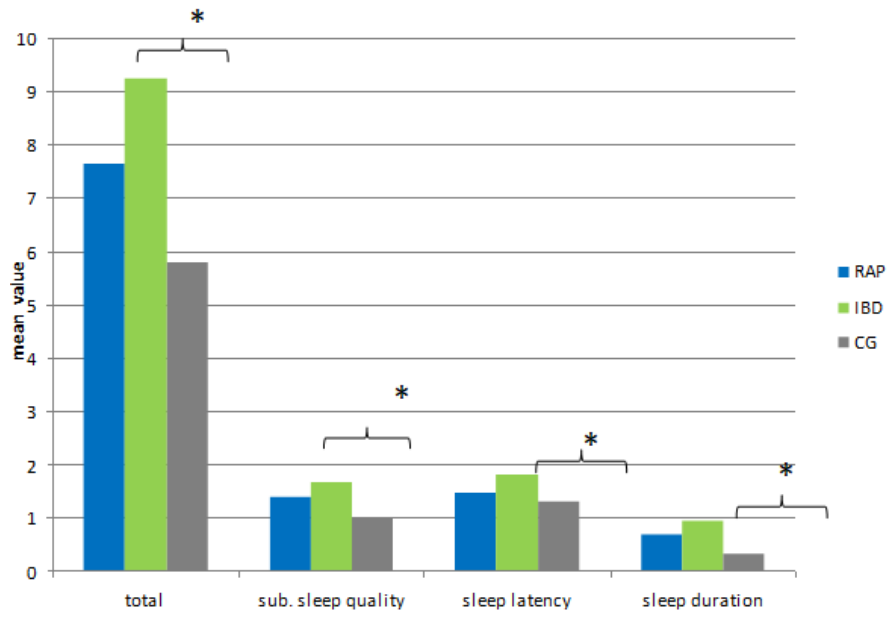

Figure 1 Mean values of the PSQI. Note: RAP = recurrent abdominal pain; IBD = irritable bowel disease; $\mathrm{CG}=$ control group; ${ }^{*} \mathrm{p}<0.05$.

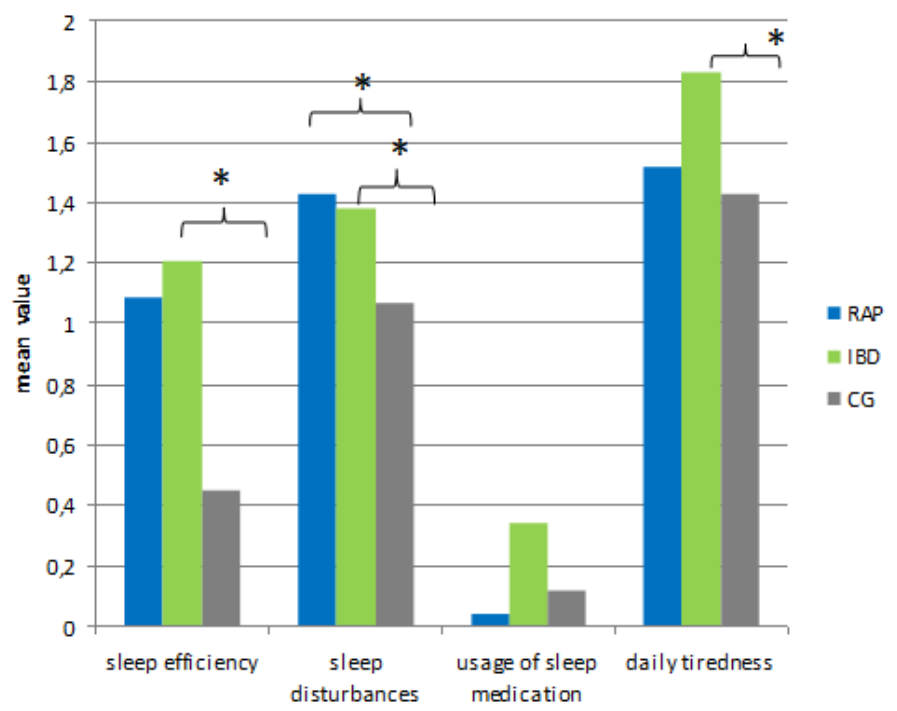

Figure 2 Mean values of the PSQI. RAP = recurrent abdominal pain; IBD = irritable bowel disease; $\mathrm{CG}=$ control group; ${ }^{*} \mathrm{p}<0.05$.

We further tested if parental reports also differed significantly regarding sleep disturbances according to the SDSC. Therefore a Multivariate analysis of variances for the sample groups was executed. Again, results indicated a significant difference between the groups, $V=0.206, \mathrm{~F}$ 
$(12,232)=2.220, p=0.001$. The results of the following Bonferroni adjusted Post-hoc tests are displayed in Figure 3. IBD patients and healthy controls differed significantly concerning the total score of the SDSC ( $p=0.002, d=-0.72$ ), as well as in 4 of 6 subscales of the SDSC: disorders of initiating and maintaining sleep $(\mathrm{p}=0.006, \mathrm{~d}=-0.67)$, disorders of arousal/nightmares $(p=0.008, d=-0.64)$, sleep wake transition disorders $(p=0.003, d=-0.70)$ and disorder of excessive somnolence ( $\mathrm{p}=0.013, \mathrm{~d}=-0.59)$ with again overall higher scores of the IBD patients. Further, the RAP group and healthy controls differed significantly concerning the total score of the SDSC $(p=0.027, d=-0.83)$ as well as regarding sleep wake transition disorders $(p=0.009, d=-1.09)$. Calculated effect sizes ranged between modest to high. In sum, sleep disturbances were more prominent in IBD patients as well as in RAP patients than in healthy controls. No significant difference was found for the subscales sleep breathing disorders and sleep hyperhidrosis.

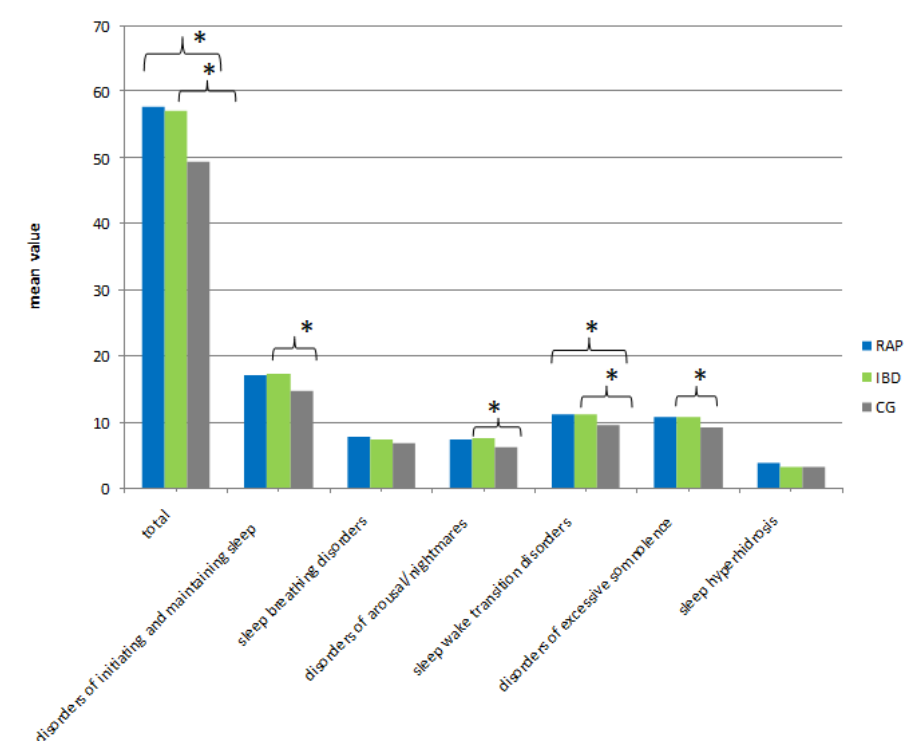

Figure 3 Mean values of the SDSC. Note: RAP = recurrent abdominal pain; $\mathrm{IBD}=$ irritable bowel disease; $\mathrm{CG}=$ control group; ${ }^{*} \mathrm{p}<0.05$.

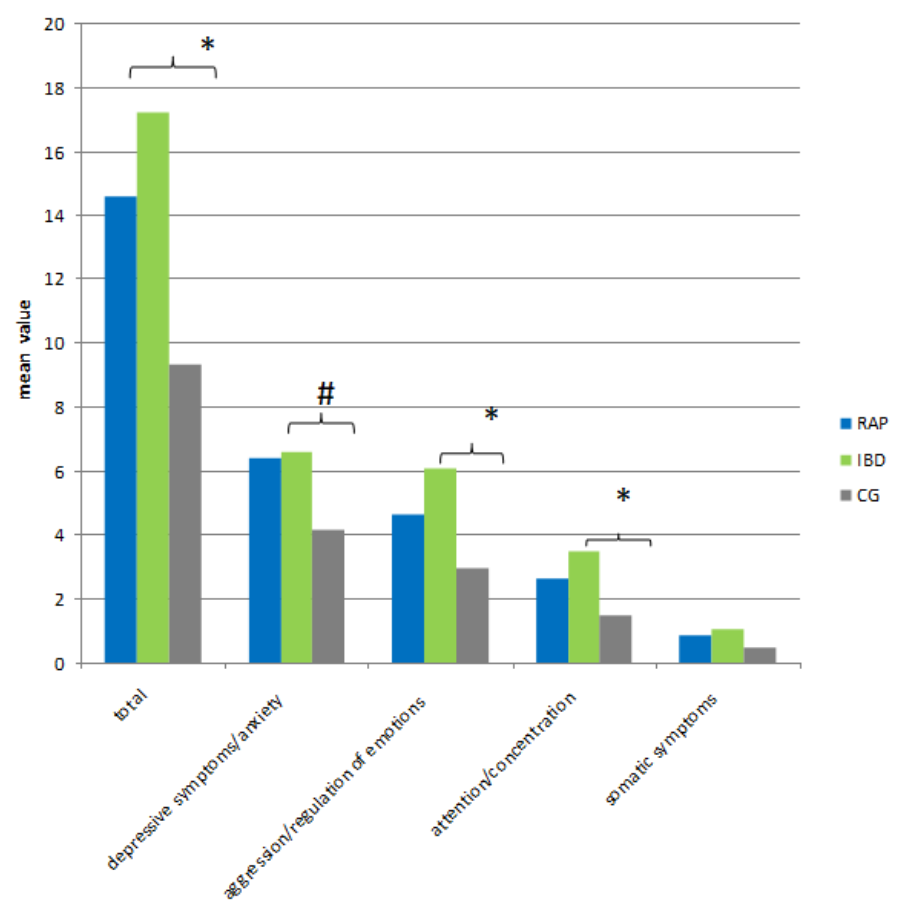

Figure 4 Mean values of the NEQ. Note: RAP = recurrent abdominal pain; IBD = irritable bowel disease; $\mathrm{CG}=$ control group; ${ }^{*} \mathrm{p}<0.10,{ }^{*} \mathrm{p}<0.05$. 
In order to compare the three sample groups concerning daytime effects of nightmares a Multivariate analysis of variances for the three sample groups was performed. Results obtained a statistically significant difference (based on the NEQ), $V=0.129, \mathrm{~F}(8,236)=2.033, \mathrm{p}=0.043$. All comparisons of the Bonferroni adjusted post hoc tests are graphically displayed in Figure 4. IBD patients and healthy controls differed significantly in regard of the total score $(p=0.009, d$ $=-0.62)$ as well as in 2 of 4 subscales of the NEQ: aggressiveness/regulation of emotions ( $p$ $=0.003, \mathrm{~d}=-0.31)$, and attention/concentration $(\mathrm{p}=0.007, \mathrm{~d}=0.10)$. The effect sizes ranged between small to modest. The results indicate that daily impairments through nightmares were more common in IBD patients than in healthy controls. For depressive symptoms/anxiety a differences was found, however it was not significant $(p=0.064, d=-0.46)$. No significant result was revealed for the subscale somatic symptoms. For the RAP group no significant effects were found.

\section{Discussion}

The aim of this study was to evaluate and compare sleep problems in adolescents and young adults with RAP respectively with IBD as well as healthy controls. In order to get a comprehensive overview self as well as parental reports were gathered. Significant differences between patient groups and healthy controls regarding a wide range of sleep related symptoms were found. Especially impaired sleep quality as well as various sleep symptoms and sleep disturbances were found. Specifically IBD adolescents suffered more often from poor sleep quality, sleep disturbances as well as daily effects of nightmares than the control group. Adolescents with RAP reported elevated scores concerning sleep disturbances compared to healthy controls. However, IBD and the RAP adolescents did not differ significantly concerning most sleep impairments.

\subsection{Sleep quality, sleep disturbances and nightmare effects in adolescents with IBD}

In detail, self-ratings concerning sleep quality measured with the PSQI, young IBD patients in the study at hand reported lower overall sleep quality, less subjective sleep quality, longer sleep latency, less sleep duration, less sleep efficiency, more sleep disturbances and more daily tiredness than healthy controls. Our results concerning sleep quality in IBD patients are in line with former studies. Especially impairments regarding overall sleep quality and sleep disturbances in IBD patients have been shown for adults as well as for children and adolescents [28,42-46]. However, Pirinen and colleagues (2010) revealed that significantly more adolescents with IBD (25\%) had sleep problems than the control group (13\%), according to parental report, whereas the adolescents themselves did not report elevated sleep difficulties [32]. The differing results might be based on the different measurements used in our study. Since Pirinen and collegues (2010) did not use a specific sleep questionnaire but rather evaluated sleep on item base our method leads to more intrinsic results concerning sleep difficulties Further our findings resemble the results of another study examining the relationship among sleep disturbances, inflammation and psychiatric dysfunction in a pediatric sample with $C D$ and depression exposing that $53 \%$ of the IBD patients had total PSQI scores resembling clinically significant sleep disturbances hence reported significantly greater sleep disruption than healthy controls [42]. Also as in our study Werkstetter and colleagues (2012) revealed that young IBD patients had a significantly prolonged sleep duration compared to controls using actigraphy [47]

Moreover, our data suggest that young IBD patients report prolonged sleep latency, a reduced sleep efficiency and more daily tiredness, hence our results give continuative insight regarding sleep disturbances in this population compared to former studies. Taken together, these results of adolescents with IBD might point towards insomnia in this population. Furthermore, a recent review emphasized that particularly sleep problems, nightmares, hypersomnia and overtiredness seem to strain these young patients suffering from IBD. In detail, especially among adolescents with IBD adequate sleep seems to be important as the disease with associated symptoms can cause distress and impair daytime functioning [48]. Similarly the current study found a significant difference between adolescents suffering from IBD and the group of healthy youngsters regarding parental ratings of sleep disturbances according to the SDSC concerning various subscales as total amount of sleep disturbances, disorders of initiating and maintaining sleep, disorders of arousal/nightmares, disorders of excessive somnolence and sleep/wake transition disorders. Based on these parental results, young IBD patients seem to have an increased risk for developing insomnia symptoms as a result of multifactorial risk factors coming along with the diseases (inflammation, pain, psychiatric dysfunctions). Hence, future 
research should aim to confirm these findings. Beyond, IBD sufferers had significantly more nightmares and more daily naps as well as significantly more overtiredness than their healthy controls according to the parents. These findings are contrary to our outcomes. However, it should be kept in mind that our data are based on adolescents self-reports. Beyond, the samples differed concerning age. The participants of the study by Pirinen and colleagues (2010) had a mean age of 15.2 years, whereas the sample at hand had a mean age of 21.72 years [32]. Further, the sleep measurements used in the two studies were different. Precisely Pirinen and colleagues (2010) investigated sleep only by single items of the child behavior checklist (CBCL) and the youth self-report (YSR) items regarding sleep [32]. Hence, the study measured sleep merely on one-item-level whereas in our study a diversity of sleep related questionnaires were implemented. Therefore, the comparability of these two studies is limited. Concerning nightmares and daytime effects of nightmares young IBD patients and healthy adolescents differed significantly again. IBD patients reported significantly more nightmares in total and suffered more from daytime impairments of their nightmares. In particular, they were more aggressive and had a higher impairment concerning emotional regulation as well as more attention and concentration difficulties than controls. This might lead to heightened stress level in young IBD patients since a high competence of emotion regulation decreases stress levels [49]. Taken those results into account, it should be noted that attention problems are associated with lower academic achievement [50]. Therefore this daytime impairment might be a further risk factor for school performance of young IBD patients. Taken together, both aspects of nightmare consequences influence daily lives of adolescents with IBD; holding the risk of worsening IBD symptoms and impairing quality of life. Further investigations should therefore focus on this topic. Precisely the specific encouragement of emotional regulation strategies as well as strategies improving attention might compensate partly the limitations due to nightmares and its daily effects in young IBD patients. In addition, young IBD patients reported more depressive symptoms and anxiety as an effect of the nightmares. However, these results showed only a tendency to significance. The nearby connection of inflammation symptoms and nightmares is in line with former studies reporting an association between nightmares and inflammation in adults [51] as well as in pediatric populations [32,48]. Beyond, it has been shown before that pain and nightmares are connected. As a study by Bruni and colleagues (1997) reported that adolescents and children with migraine or tension headache report significantly more nightmares than controls [52]. Also the working group of Miller reported that children with migraine suffered significantly more from parasomnias than healthy controls [53].

However, the study at hand is the first to our knowledge investigating the daytime effects of nightmares in a pediatric sample with chronic abdominal pain, showing that nightmares in young IBD patients do impair their daily lives. Our results demonstrate that nightmares in young IBD patients are associated with daytime impairments regarding emotion regulation and attention problems.

\subsection{Sleep quality, sleep disturbances and nightmare effects in ado- lescents with RAP}

The results of our study showed a significant difference between adolescents with RAP and healthy controls concerning sleep quality. According to the self-ratings RAP patients differed significantly regarding sleep disturbances. In addition, parental ratings revealed a significant difference in the total amount of sleep disorders as well as for problems falling and/or maintaining sleep. No significant differences could be revealed regarding the effects of nightmares. Our findings concerning sleep disorders in adolescents with RAP reflect the results of a former study by Huntley and colleagues (2007) where young RAP patients reported an increased problems initiating and maintaining sleep, distressing thoughts related to pain or night-time fears as well as self-ratings of poor sleep quality compared to a healthy control group [10]. Similar results were described by Schurman and colleagues (2012) [54]. In their study children and adolescents, aged 8-17 years, with chronic abdominal pain had clinically elevated scores on at least one subscale of the SDSC. Therefore, results lead to the conclusion that especially sleep disturbances are a burden for those adolescents with RAP whereas in young IBD patients the problems are more diverse.

\subsection{Comparison of adolescents with IBD and RAP}

Our study showed that both pain groups differed significantly concerning various sleep related problems in comparison to healthy controls. However, both groups, IBD and RAP differed mostly not concerning sleep parameters showing that both are highly impaired. This non-difference is in line with former studies addressing adults with IBD and irritable bowel 
diseases (IBS), with no significant differences concerning sleep difficulties [30,54]. This leads to the conclusion that sleep problems of IBD and RAP patients seem to be comparable regarding sleep impairments.

However concerning the comparison to the healthy controls, IBD patients reported more significant differences regarding sleep difficulties than adolescents with RAP. This fact could be explained by the different causes of the diseases. Whereas RAP is a disease without organic basis [55,56], in IBD physiological factors play an important role [57], hence in IBD other factors must be taken into account regarding sleep difficulties.

All in all, the results at hand indicate that young IBD patients are more impaired concerning sleep problems than youngsters with RAP in comparison to healthy controls.

\section{Strengths and limitations}

The study at hand has some limitations. First, it was an online survey based on subjective measurements concerning sleep as well as pain diseases. In future investigations objective measurements should be implemented in order to confirm subjective data. However, we asked for disorders based on pediatrician's diagnosis. Further, the mean age of the sample was with around 20 years higher than expected, thus the comparability to former investigations addressing children or adolescents is limited, since in most previous data mean age was around 15 years or younger $[10,32,42,58]$. Beyond, in our study the sample groups differed regarding mean age with a significant difference of the IBD adolescents compared to the RAP group as well as the healthy controls. Moreover, concerning the IBD, adolescents with $\mathrm{CD}$ were more represented than those suffering from UC. Besides, in the study at hand more female participants took part. Future investigations should mind a balanced sample concerning IBD diagnosis, gender as well as a comparable mean age to previous studies. Additionally, duration and severity of the diseases should be taken into account. Further, complications or medication use should be considered since those factors might influence the course of the disease as well as sleep related conditions.

Strength of the current investigation is the examination and comparison of young RAP and IBD patients concerning the impact of the particular disease on sleep as well as contrasting those groups to healthy controls; filling an existing gap in this area of research. Further we accumulated self as well as parental ratings in order to provide a holistic survey of sleep problems. Beyond, this study implemented various sleep related questionnaires.

\section{Conclusion and further directives}

Our study was able to show that in adolescents with IBD and RAP sleep quality is impaired and both sample groups have a higher rate of sleep disturbances. Moreover data showed that especially young IBD patients suffer from daily effects from nightmares. This study at hand was the first to compare adolescents with IBD and RAP regarding sleep difficulties, revealing a worse and impaired sleep of young IBD patients. Additionally, compared to earlier research a broader range of sleep disorders was investigated (i.e. nightmares), giving more detailed information of specific sleep problems.

\section{Ethics statement}

The study was approved by the Ethics Committee of the University (Number: EUB-2017-019). Participants gave their informed consent and were informed that participation was voluntary. The study follows the latest version of the Declaration of Helsinki.

\section{Availability of data and materials}

The datasets used and analyzed during the current study are used for further analysis and are available from corresponding author on reasonable request.

\section{Authors' contributions}

Ann-Kristin Manhart collected, analyzed and interpreted the data and has been involved in drafting the manuscript; Angelika A. Schlarb had the idea of the study, recommended the methods, supervised the study, made substantial contributions to the analysis and interpretation of the data and revised the manuscript critically. All authors read and approved the final manuscript. 


\section{Conflict of interest}

The authors declare that they have no competing interests.

\section{Acknowledgements}

We acknowledge support for distributing the online link and the data collection by the Gesellschaft für Pädiatrische Gastroenterologie und Ernährung e.V. as well as for Article Processing Charge by the Deutsche Forschungsgemeinschaft and the Open Access Publication Fund of Bielefeld University.

\section{References}

[1] Scharff L. Recurrent abdominal pain in children: a review of psychological factors and treatment. Clinical psychology review, 1997, 17(2): 145-166. https://doi.org/10.1016/S0272-7358(96)00001-3

[2] Schlarb AA, Gulewitsch MD, Bock genannt Kasten I, et al. Recurrent abdominal pain in children and adolescents - a survey among paediatricians. GMS Psycho-Social-Medicine. 2011, 8: 1-7.

[3] Rasquin A, Di Lorenzo C, Forbes D, et al. Childhood functional gastrointestinal disorders: child / adolescent. Gastroenterology, 2006, 130(5): 1527-1537. https://doi.org/10.1053/j.gastro.2005.08.063

[4] Abu-Arafeh I and Russell G. Prevalence and clinical features of abdominal migraine compared with those of migraine headache. Archives of disease in childhood, 1995, 72(5): 413-417. https://doi.org/10.1136/adc.72.5.413

[5] Hyams JS, Burke G, Davis PM, et al. Abdominal pain and irritable bowel syndrome in adolescents: a community-based study. The Journal of pediatrics, 1996, 129(2): 220-226. https://doi.org/10.1016/S0022-3476(96)70246-9

[6] Youssef NN, Atienza K, Langseder AL, et al. Chronic abdominal pain and depressive symptoms: analysis of the national longitudinal study of adolescent health. Clinical Gastroenterology and Hepatology, 2008, 6(3): 329-332. https://doi.org/10.1016/j.cgh.2007.12.019

[7] Saps M, Seshadri R, Sztainberg M, et al. A prospective school-based study of abdominal pain and other common somatic complaints in children. The Journal of pediatrics, 2009, 154(3): 322-326. https://doi.org/10.1016/j.jpeds.2008.09.047

[8] Youssef NN, Murphy TG, Langseder AL, et al. Quality of life for children with functional abdominal pain: a comparison study of patients' and parents' perceptions. Pediatrics, 2006, 117(1): 54-59. https://doi.org/10.1542/peds.2005-0114

[9] Drossman DA. Gastrointestinal illness and the biopsychosocial model. Psychosomatic medicine 1998, 60(3): 258-267. https://doi.org/10.1097/00006842-199805000-00007

[10] Huntley ED, Campo JV, Dahl RE, et al. Sleep characteristics of Youth with Functional Abdominal Pain and a Healthy Comparison Group. J of Pediatr. Psychology, 2007, 32(8): 938-949. https://doi.org/10.1093/jpepsy/jsm032

[11] Haim A, Pillar G, Pecht A, et al. Sleep patterns in children and adolescents with functional recurrent abdominal pain: objective versus subjective assessment. Acta Paediatrics, 2004, 93(5): 677-680. https://doi.org/10.1111/j.1651-2227.2004.tb02996.x

[12] Kim HJ. Importance of sleep quality in functional abdominal pain disorder in pediatric patients. Sleep and Biological Rhythms, 2021: 1-5. https://doi.org/10.1007/s41105-021-00342-9

[13] Wang B, Duan R and Liping D. Prevalence of sleep disorder in irritable bowel syndrome: A systematic review with meta-analysis. Saudi Journal of Gastroenterology, 2018, 24(3): 141-150. https://doi.org/10.4103/sjg.SJG_603_17

[14] Pavlova M, Ference J, Hancock M, et al. Disentangeling the Sleep Pain Relationship in Pediatric Chronic Pain: The Mediating Role of Internalizing Mental Health Symptoms. Pain Research and Management, 2017. https://doi.org/10.1155/2017/1586921

[15] Abraham C and Cho JH. Inflammatory bowel disease. New England Journal of Medicine, 2009, 361 2066-2078. https://doi.org/10.1056/NEJMra0804647

[16] Loftus EV. Clinical epidemiology of inflammatory bowel disease: Incidence, Prevalence, and Environmental Influences. Gastroenterol, 2004, 126: 1504-1517. https://doi.org/10.1053/j.gastro.2004.01.063

[17] Zimmerman J. Extraintestinal symptoms in irritable bowel syndrome and inflammatory bowel diseases: nature, severity, and relationship to gastrointestinal symptoms. Dig Dis Science, 2003, 48(4): 743 749.

https://doi.org/10.1023/A:1022840910283 
[18] Guan Q. A Comprehensive Review and Update on the Pathogenesis of Inflammatory Bowel Disease. Journal of immunology research, 2019, 2019: 7247238 . https://doi.org/10.1155/2019/7247238

[19] Benchimol EI, Fortinsky KJ, Gozdyra P, et al. Epidemiology of pediatric inflammatory bowel disease: a systematic review of international trends. Inflammatory Bowel Diseases, 2011, 17(1): 423-439. https://doi.org/10.1002/ibd.21349

[20] Guilfoyle SM, Gray WN, Herzer-Maddux M, et al. Parenting stress predicts depressive symptoms in adolescents with inflammatory bowel disease. European Journal of Gastroenterology \& Hepatology, 2014, 26(9): 964-971. https://doi.org/10.1097/MEG.0000000000000149

[21] Amre DK, Lu SE, Costea F, et al. Utility of serological markers in predicting the early occurrence of complications and surgery in pediatric Crohn's disease patients. American Journal of Gastroenterology, 2006, 101(3): 645-652. https://doi.org/10.1111/j.1572-0241.2006.00468.x

[22] Bousvaros A, Sylvester F, Kugathasan S, et al. Challenges in pediatric inflammatory bowel disease. Inflammatory Bowel Diseases, 2006, 12(9): 885-913. https://doi.org/10.1097/01.mib.0000228358.25364.8b

[23] Dubinsky MC, Lin YC, Dutridge D, et al. Serum immune responses predict rapid disease progression among children with Crohn's disease: immune responses predict disease progression. American Journal of Gastroenterology, 2006, 101(2): 360-367. https://doi.org/10.1111/j.1572-0241.2006.00456.x

[24] Engelmann G, Erhard D, Petersen M, et al. Health-related quality of life in adolescents with inflammatory bowel disease depends on disease activity and psychiatric comorbidity. Child Psychiatry \& Human Development, 2015, 46(2): 300-307. https://doi.org/10.1007/s10578-014-0471-5

[25] Starpersma L, van den Brink G, van der Ende J, et al. Illness Perception and Depression Are Associated with Health-Related Quality of Life in Youth with Inflammatory Bowel Disease. International Journal of Behavioral Medicine, 2019, 26: 415-426. https://doi.org/10.1007/s12529-019-09791-6

[26] Gamwell KL, Baudino MN, Bakula DM, et al. Perceived Illness Stigma. Thwarted Belongingness, and Depressive Symptoms in Youth With Inflammatory Bowel Disease (IBD). Inflamm Bowel Disease, 2018, 24(5): 960-965. https://doi.org/10.1093/ibd/izy011

[27] Selemon LD. A role for synaptic plasticity in the adolescent development of executive function. Translational Psychiatry, 2013, 3: 1-9. https://doi.org/10.1038/tp.2013.7

[28] Ananthakrishnan AN, Long MD, Martin CF, et al. Sleep disturbance and risk of active disease in patients with Crohn's disease and ulcerative colitis. Clinical Gastroenterology and Hepatology, 2013, 11(8): 965-971. https://doi.org/10.1016/j.cgh.2013.01.021

[29] Sobolewska-Wlodarczyk A, Wlodarczyk M, Banaski J, et al. Sleep disturbances and disease activity in adult patients with inflammatory bowel disease. Journal of Physiology and Pharmacology, 2018, 69(3): 423-428. https://doi.org/10.26402/jpp.2018.3.09

[30] Ranjbaran Z, Keefer L, Stepanski E, et al. The relevance of sleep abnormalities to chronic inflammatory conditions. Inflammation Research, 2007, 56(2): 51-57. https://doi.org/10.1007/s00011-006-6067-1

[31] Schredl M, Fricke-Oerkermann L, Mitschke A, et al. Longitudinal Study of Nightmares in Children: Stability and Effect of Emotional Symptoms. Child Psychiatry \& Human Development, 2009, 40(3): 439-449. https://doi.org/10.1007/s10578-009-0136-y

[32] Pirinen T, Kolho KL, Simola P, et al. Parent and Self-Report of Sleep-Problems and Daytime Tiredness Among Adolescents With Inflammatory Bowel Disease and Their Population-Based Controls. Sleep 2010, 33(11): 1487-1493. https://doi.org/10.1093/sleep/33.11.1487

[33] QuestBack AG. Unipark. EFS Survey Software, 2015.

[34] Buysse DJ, Reynolds CF, Monk TH, et al. The Pittsburgh Sleep Quality Index: A new instrument for psychiatric practice and research. Psychiatry Research, 1989, 28(2): 193-213. https://doi.org/10.1016/0165-1781(89)90047-4

[35] Mollayeva T, Thurairajah P, Burton K, et al. The Pittsburgh sleep quality index as a screening tool for sleep dysfunction in clinical and non-clinical samples: a systematic review and meta-analysis. Sleep medicine reviews, 2016, 25: 52-73. https://doi.org/10.1016/j.smrv.2015.01.009

[36] Chiu HY, Chang LY, Hsieh YJ, et al. A meta-analysis of diagnostic accuracy of three screening tools for insomnia. Journal of psychosomatic research, 2016, 87: 85-92. https://doi.org/10.1016/j.jpsychores.2016.06.010

[37] Wittchen HU, Krause P, Höfler M, et al. NISAS-2000-die "Nationwide Insomnia Screening and Awareness Study", 2001.

[38] Backhaus J, Junghanns K, Broocks A, et al. Test-retest reliability and validity of the Pittsburgh Sleep Quality Index in primary insomnia. Journal of Psychosomatic Research, 2002, 53(3): 737-740. https://doi.org/10.1016/S0022-3999(02)00330-6 
[39] Schlarb AA, Zschoche M and Schredl M. Der Nightmare Effects Questionnaire (NEQ). Pilotstudie $\mathrm{zu}$ ersten psychometrischen Kennwerten bei Jugendlichen und jungen Erwachsenen. Somnologie, 2016, 20(4): 251-257. https://doi.org/10.1007/s11818-016-0086-0

[40] Bruni O, Ottaviano S, Guidetti V, et al. The Sleep Disturbance Scale for Children (SDSC) Construct ion and validation of an instrument to evaluate sleep disturbances in childhood and adolescence. Journal of sleep research, 1996, 5(4): 251-261. https://doi.org/10.1111/j.1365-2869.1996.00251.x

[41] Cohen J. Statistical Power Analysis for the Behavioral Sciences (2. Aufl.). London, UK: Taylor \& Francis, 1988.

[42] Benhayon D, Youk A, McCarthy FN, et al. Characterization of relations among sleep, inflammation, and psychiatric dysfunction in depressed youth with Crohn's disease. Journal of Pediatric Gastroenterology \& Nutrition, 2013, 57(3): 335-342. https://doi.org/10.1097/MPG.0b013e31829641df

[43] Ali T, Madhoun MF, Orr WC, et al. Assessment of the relationship between quality of sleep and disease activity in inflammatory bowel disease patients. Inflammatory Bowel Diseases, 2013, 19(11): $2440-2443$ https://doi.org/10.1097/MIB.0b013e3182a0ea54

[44] Uemura R, Fujiwara Y, Iwakura N, et al. Sleep disturbances in Japanese patients with inflammatory bowel disease and their impact on disease flare. Springer Plus, 2016, 5(1): 1792. https://doi.org/10.1186/s40064-016-3408-6

[45] Forrest CB, Forrest KD, Clegg JL, et al. Establishing the content validity of PROMIS Peditric pain interference, fatigue, sleep disturbance, and sleep-related impairment measures in children with chronic kidney disease and Crohn's disease. Journal of Patient-Reported Outcomes, 2020, 4: 11. https://doi.org/10.1186/s41687-020-0178-2

[46] Jarasvaraparn C, Zlomke K, Vann NC, et al. The realtionship between sleep disturbance, and disease activity in pediatric patients with inflammatory bowel disease. Journal of Pediatric Gastroenterology and Nutrition, 2019, 68(2): 237-243. https://doi.org/10.1097/MPG.0000000000002156

[47] Werkstetter KJ, Ullrich J, Schatz SB, et al. Lean body mass, physical activity and quality of life in paediatric patients with inflammatory bowel disease and in healthy controls. Journal of Crohn's and Colitis, 2012, 6(6): 665-673. https://doi.org/10.1016/j.crohns.2011.11.017

[48] Manhart AK, Hellmann S, Hamelmann E, et al. The association of sleep with inflammatory bowel disease in children and adolescents. Somnologie, 2016, 20(3): 212-218. https://doi.org/10.1007/s11818-016-0067-3

[49] Wirtz PH, von Känel R, Mohiyeddini C, et al. Low social support and poor emotional regulation are associated with increased stress hormone reactivity to mental stress in systemic hypertension. The Journal of Clinical Endocrinology \& Metabolism, 2006, 91(10): 3857-3865. https://doi.org/10.1210/jc.2005-2586

[50] Barriga AQ, Doran JW, Newell SB, et al. Relationships between problem behaviors and academic achievement in adolescents: The unique role of attention problems. Journal of Emotional and Behavioral disorders, 2002, 10(4): 233-240. https://doi.org/10.1177/10634266020100040501

[51] Purabdollah M. Relationship between Sleep, Pain and Inflammatory Markers in Patients with Rheumatoid Arthritis. Journal of Caring Sciences, 2017, 6(3): 249 https://doi.org/10.15171/jcs.2017.024

[52] Bruni O, Fabrizi P, Ottaviano S, et al. Prevalence of sleep disorders in childhood and adolescence with headache: a case-control study. Cephalalgia, 1997, 17(4): 492-498. https://doi.org/10.1046/j.1468-2982.1997.1704492.x

[53] Miller VA, Palermo TM, Powers SW, et al. Migraine headaches and sleep disturbances in children. Headache: The Journal of Head and Face Pain, 2003, 43(4): 362-368. https://doi.org/10.1046/j.1526-4610.2003.03071.x

[54] Schurman JV, Friesen CA, Dai H, et al. Sleep problems and functional disability in children with functional gastrointestinal disorders: an examination of the potential mediating effects of physical and emotional symptoms. BMC gastroenterology, 2012, 12(1): 142. https://doi.org/10.1186/1471-230X-12-142

[55] Ernst AR, Routh DK and Harper DC. (1984). Abdominal pain in children and symptoms of somatization disorder. Journal of Pediatric Psychology, 1984, 9(1): 77-86. https://doi.org/10.1093/jpepsy/9.1.77

[56] Routh DK, Ernst AR and Harper DC. Recurrent abdominal pain in children and somatization disorder. In D. K. Routh (Ed.), Handbook of pediatric psychology (pp. 492-504). New York, NY, US: Guilford Press, 1988.

[57] Baumgart DC and Sandborn WJ. Inflammatory bowel disease: clinical aspects and established and evolving therapies. The Lancet, 2007, 369(9573): 1641-1657. https://doi.org/10.1016/S0140-6736(07)60751-X

[58] Pirinen T, Kolho KL, Ashorn M, et al. Sleep and Emotional and Behavioral Symptoms in Adolescents with Inflammatory Bowel Disease. Sleep Disorders, 2014, 2014: 379450. https://doi.org/10.1155/2014/379450 